\title{
Crystal Structure: Reciprocal Space Methods for Carry out the Structure Solution from Powder Data
}

\author{
Mbark Ait Mouha*, Dounia Tlamsamani, Khalid Yamni \\ Department of Chemistry, Faculty of Sciences, Moulay Ismail University, Meknes, Morocco \\ Email address: \\ aitmouha.m@gmail.com (M. A. Mouha) \\ ${ }^{*}$ Corresponding author \\ To cite this article: \\ Mbark Ait Mouha, Dounia Tlamsamani, Khalid Yamni. Crystal Structure: Reciprocal Space Methods for Carry out the Structure Solution \\ from Powder Data. International Journal of Materials Science and Applications. Vol. 10, No. 2, 2021, pp. 25-29. \\ doi: $10.11648 /$ j.ijmsa.20211002.11
}

Received: February 22, 2021; Accepted: March 10, 2021; Published: April 1, 2021

\begin{abstract}
It is a relatively easy task to the solution of the so-called phase problem in crystallography, by applying ab initio phasing methods for the efficiency of structure solution from single-crystal data. Their effective application to powder x-ray diffraction data is still a real challenge unless the size of the structure is moderate. The percentage of principal success hinges on a number of factors; included are the quality of the experimental pattern, the success of the pattern-decomposition programs, the quality of the extracted structure-factor from the experimental pattern via the Le Bail or Pawley methods, the normalization of structure-factor process, the experimental resolution and the straightforward of the phasing process. This paper aims at providing an overall overview of the reciprocal space RS methods (ab initio phasing methods of crystal structure) as well as the direct methods, Patterson function and maximum entropy methods. This paper will also describe the factors affecting phasing by reciprocal space methods and the limitation of reciprocal space methods. Those are available for carry out the structure solution, in order to provide a clear theoretical account, experimental practice and computing approaches regarding and describe an outline of the solution process of phase problem by powder X-ray diffraction, leads to the best structure solution using practical examples.
\end{abstract}

Keywords: Powder Data, Data Quality, Reciprocal Space, Structure Solution, Ab initio Phasing Methods, Pattern Decomposition, Structure Factor

\section{Introduction}

Structure solution from powder x-ray diffraction data (PXRD) is considered to be a real challenge in crystallography. The determination of the unit cell parameters is the primary step in structure solution from PXRD data, the cell-characterization process is also called 'indexing'. This later, by autoindexing methods, is normally put in to action, for which a number of computer programs are available; ITO [1], TREOR [2], DICVOL [3] and NTREOR09 [4].

The indexing process is the determination of $\mathrm{d}_{\mathrm{hkl}}$ interplanar spacing, smoothed by high quality data and the determination of the unit cell parameters. The second steps, when first step has been accomplished, is the decomposition of the powder pattern in to integrated intensities. These later are connected with each reflection in the experimental profile The peak overlapping in powder diffraction pattern leads to the amount of experimental information: small number of structure amplitudes (several hundred) that are measured in the powder experiment compared with the several thousand of a single crystal experiment. Such problems define all the steps of the solution process.

For overcoming the peak overlapping problem, will have recourse to two methods for extraction of structure factor amplitudes: The Pawley and Le Bail methods, both were derived from the Rietveld method [5]. Special software packages have been developed. as well as EXTRA (Le Bail algorithm [6]) and ALLHKL (Pawley algorithm [7]).

We will assume that the unit cell dimensions are supposed to be calculated, the space group is determined and the structure-factor moduli extracted from the experimental powder diffraction data are associated with each reflection.

The aim of this paper is to provide, in particular an overview of different methods operate in reciprocal space 
which are available for structure solution from powder data using ab initio phasing methods.

\section{The Reciprocal Space (RS) Methods}

The RS methods for structure solution is based on the following steps: The identification of the unit cell parameters, determination of the space group, extraction of structure factor amplitudes, identification of the phase of structure factors, Fourier synthesis. After sample preparation and diffraction data collection, only the structure factor moduli are extracted while the relative phases are hidden, to the solution of the phase problem. Ab initio phasing methods have developed to the determination of the phase of structure factors (phase problem). These are most known as direct [8], Patterson [9] and maximum entropy methods [10].

\subsection{Direct Methods (DM)}

Direct Methods do not need prior information. Their aims is explained by the name: to derive directly the phase of structure factors.

DM theory is grounded up on three main hypotheses [11]:

1) The electron density $\rho$ (r) is positive everywhere: positivity;

2) The $\rho(r)$ is concentrated around the nuclei: atomicity;

3) The uniform and independent distribution of atoms in the unit cell.

The phasing process by DM carries out the following principal steps [11]:

(1) Normalization: In order to overcome the dependence of $\mathrm{F}_{\mathrm{hkl}}$ on the overall isotropic displacement factor $\mathrm{B}$, on the scale factor $\mathrm{k}$ and on data resolution, $\mathrm{k}$ and $\mathrm{B}$ can be estimated by the Wilson plot [12]. Direct methods work with normalized structure factors $\mathrm{E}_{\mathrm{hkl}}$. Their distribution can help to space-group determination and the strong reflections (largest $\left|\mathrm{E}_{\mathrm{hkl}}\right|$ ) play a principal role in the efficiency of phasing process.

(2) Estimate of structure invariants: After normalization, it is important to consider that the normalized structure factor denotes a novel origin $\mathrm{x}_{0}$, while the modulus remains unchanged $\left|\mathrm{E}_{\mathrm{hkl}}\right|=\left|\mathrm{E}_{\mathrm{hkl}}^{\prime}\right|$ (it is structure invariant). A more general concept and theory, has been formulated by Giacovazzo [13, 14, 8, 15, 16]. The main structure invariants (triplet invariants were first given by Cochran [17]) are calculated by considering the set of strong reflections (largest $\left.\left|E_{h k l}\right|\right)$. The $P_{10}$ conditional probability distribution [18] is more powerful than Cochran's formula because use of strong and weak $\left|E_{h k l}\right|$ values. The $E_{h k l}$ values are assessed and actively used in the phasing process.

(3) Phasing: This process calculates the phases of strong reflections, or assignment of initial phases to all of the normalized reflections and the generation of new or refined phases, by exploiting triplet relationships. In general, only the reflections having $|\mathrm{E}|>1$ are phased using the tangent formula [19]. The phasing process offers a number of possible sets of phases. They are classified by figures of merit (FOM sand / or CFOM) in order to estimate their reliability. A new figure of merit, an (AMBFOM), has been proposed [20], joined with a special decomposition procedure $[21,22]$. This later in tends to select a number of models to be a refinement procedure.

(4) E- map calculation: After the determination of phases of reflections. The best phase calculated by phasing process is used for calculating an $\rho$ (r) map via a Fourier synthesis for which the coefficients are the $|\mathrm{E}|$ values. The Fourier synthesis gives the direct image of $\rho$ (r) distribution, matches positions of atoms in the unit cell. By taking in to account the cell content, a chemical interpretation of the peak positions is performed. The quality of structural model is improved by calculating the coefficient of reliability $\mathrm{R}_{\mathrm{F}}$, the best structural model corresponding to lowest $R_{F}$ value is directly and carefully chosen.

\subsection{Patterson Methods (PM)}

The first approach to structure determination was developed by Patterson [9], who proposed an expression for function $\mathrm{P}(\mathrm{u})$ (Its name is due to Patterson) in terms of Fourier synthesis of the observed $\left|F_{h}\right|^{2}$ values as coefficients and thus no phasing is needed.

For interpreting the Patterson map, the most approach is based on the use of Harker sections [23] which comprise interatomic vectors. By using the Harker sections, it is possible to delimit the heavy-atom positions from which the light atoms can be situated by Fourier synthesis the observed $\left|\mathrm{F}_{\mathrm{h}}\right|^{2}$ values and the phases calculated from the heavy atoms. The use of the symmetry minimum function SMF [24] is more effective than the manual inspection of Harker sections [25].

The Patterson-function has also been used to increase the quality of the extracted integrated intensities of reflections [26]. Alternatively, the FIPS (fast iterative Patterson squaring) procedure can be used [27].

\subsection{Maximum Entropy Methods}

This approach was devised by Bricogne [10] who gave a general concept and theory for using maximum entropy (ME) for structure solution from powder diffraction data. The aim of ME is de calculation of a discrete probability distribution by the maximization of their entropy using strong unphased reflections with permuted phase values. a number of phase sets are estimated and uses to constrained entropy maximization [11]. In the field of bad data quality, ME methods were found to be more than direct methods [28]. ME is embodied in the computer program MICE [29]. This later has only been practice to a number of powder x-ray diffraction data [30].

There was a number of important reasons for the efficiency of structure solution from x-ray powderdiffraction data include the quality of diffraction pattern, the experimental resolution [31], the quality of the extracted structure-factor moduli and the accessibility of software packages [11]. 


\section{Examples of Structure Determination from Powder Diffraction Data}

In practice we place our focus on examples of structure solution of organic compounds by means of reciprocal space techniques, EXPO [32], is uniquely able to carry out the complete structure determination process: indexing, spacegroup determination, solving the structure by DM or DS techniques, and finally refining by the Rietveld method.

Two examples of organic compounds structure solved by using the Software packages EXPO [32, 33]:

Example 1: 2-Mercaptobenzoic acid $\mathrm{C}_{7} \mathrm{H}_{6} \mathrm{O}_{2} \mathrm{~S}$

This concerns the 2-Mercaptobenzoicacid structure [34]:

1. wavelength $=1.54056 \AA$,

2. 2-theta experimental range (5-65)
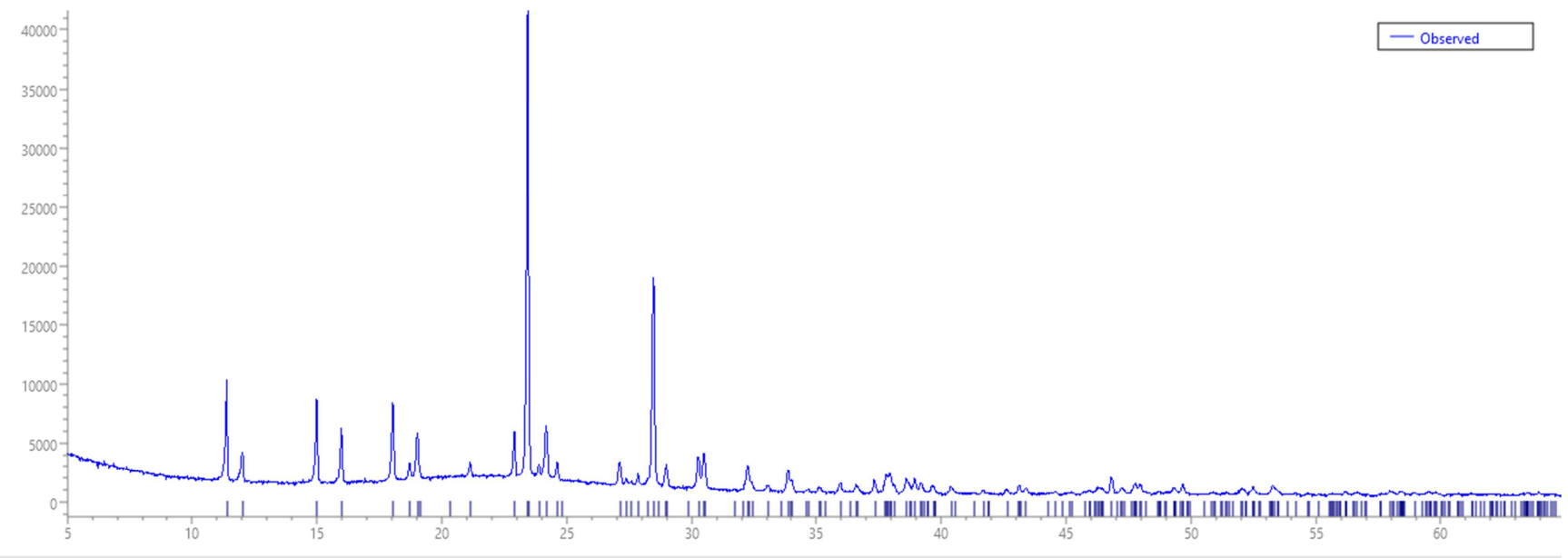

Figure 1. Experimental pattern of 2-Mercaptobenzoic acid.

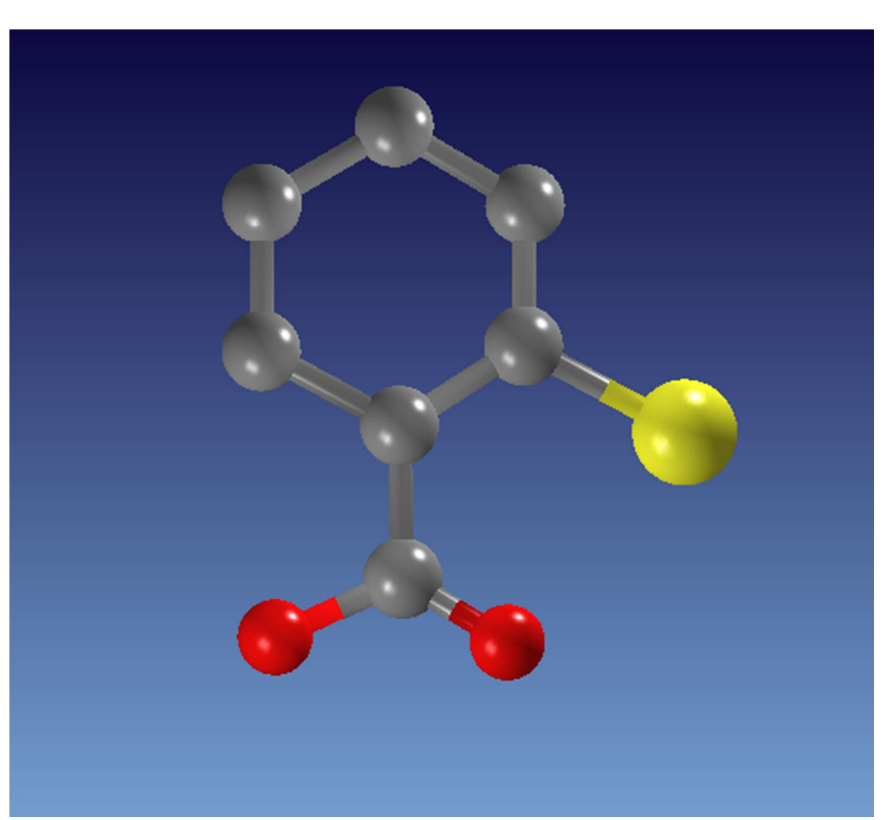

Figure 2. Crystal structure of example 1 visualized on JAV Molecular Viewer.
3. Cell parameters: $\mathrm{a}=7.885, \mathrm{~b}=5.974, \mathrm{c}=14.948$,

4. alpha $=90.000$, beta $=100.497$, gamma $=90.000$

5. 4 molecules of $\mathrm{C}_{7} \mathrm{H}_{6} \mathrm{O}_{2} \mathrm{~S}$ in the unit cell

6. 245 reflections

7. Space Group p 21/c

8. Experimental resolution $=1.43 \AA$

Giacovazzo indicates that the nominal resolution which a small organic or inorganic molecule structure cannot be deciphered ab initio is $\mathrm{d}_{\min } \approx 1.4 \AA$ [35]. whose experimental pattern is shown in Figure 1. 85 largest E-values $(|E|>1)$ are phased by DM using the tangent formula [19]. These later generate several trials; the largest CFOM [11] is automatically selected. The best solution (lowest $R_{F}$ value) will be selected, refined by the Rietveld method and displayed on JAV Molecular as shown in Figure 2.

\section{Example 2: Cimetidine $\mathrm{C}_{10} \mathrm{H}_{16} \mathrm{~N}_{6} \mathrm{~S}$}

The crystal structure of Cimetidine [36] is monoclinic. Whose experimental pattern is shown in Figure 3.

1 . wavelength $=1.52904 \AA$,

2. 2-theta experimental range $(8-85)$

3. Cell parameters: $\mathrm{a}=10.394 \mathrm{~b}=18.818 \mathrm{c}=6.824$

4. alpha $=90.000$ beta $=106.437$ gamma $=90.000$

5. 4 molecules of $\mathrm{C}_{10} \mathrm{H}_{16} \mathrm{~N}_{6} \mathrm{~S}$ in the unit cell

6. 924 reflections

7. Space Group p 21/a

8. Experimental resolution $=1.13 \AA$

The structure factors were obtained by EXTRA [5], program for pattern decomposition. The normalization process generate 268 strongest reflections having $|\mathrm{E}|>1.08$. These sets of strong reflections are phased using the tangent formula [19].

The crystal structure was phasing by DM followed by Fourier recycling and Fourier syntheses. The final result with the largest CFOM and lowest $\mathrm{R}_{\mathrm{F}}$ value will be visualized on JAV Molecular as shown in Figure 4. 


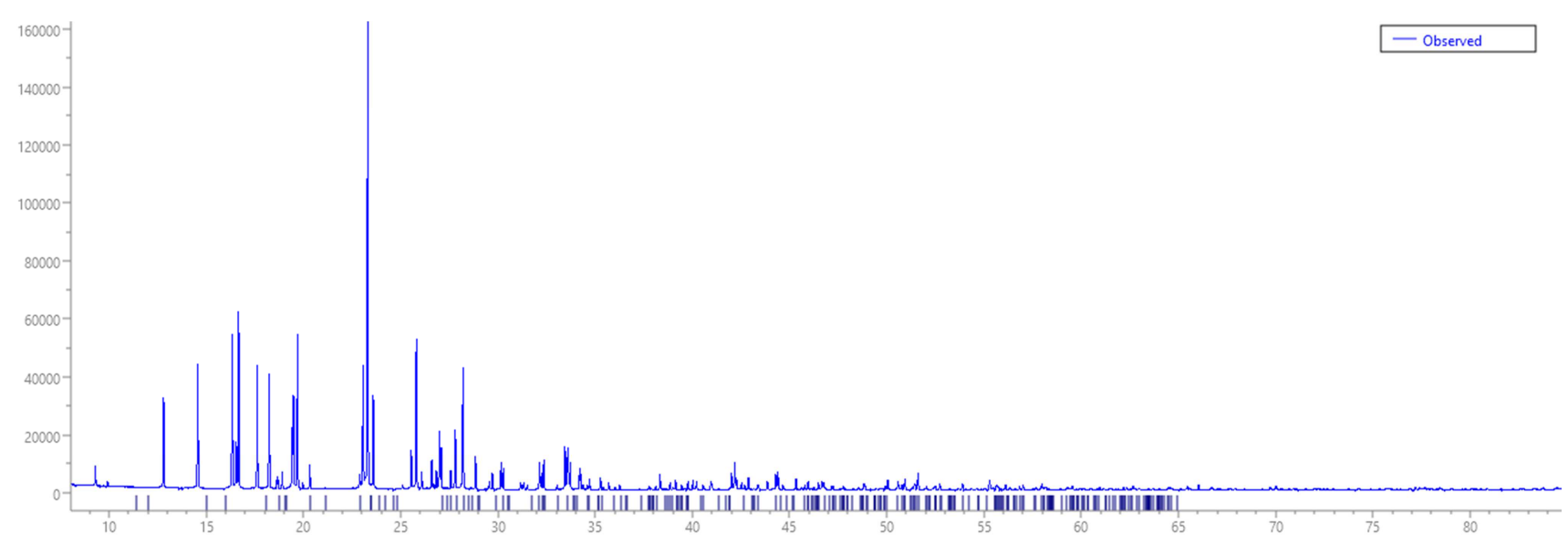

Figure 3. Experimental patternof cimetidine.

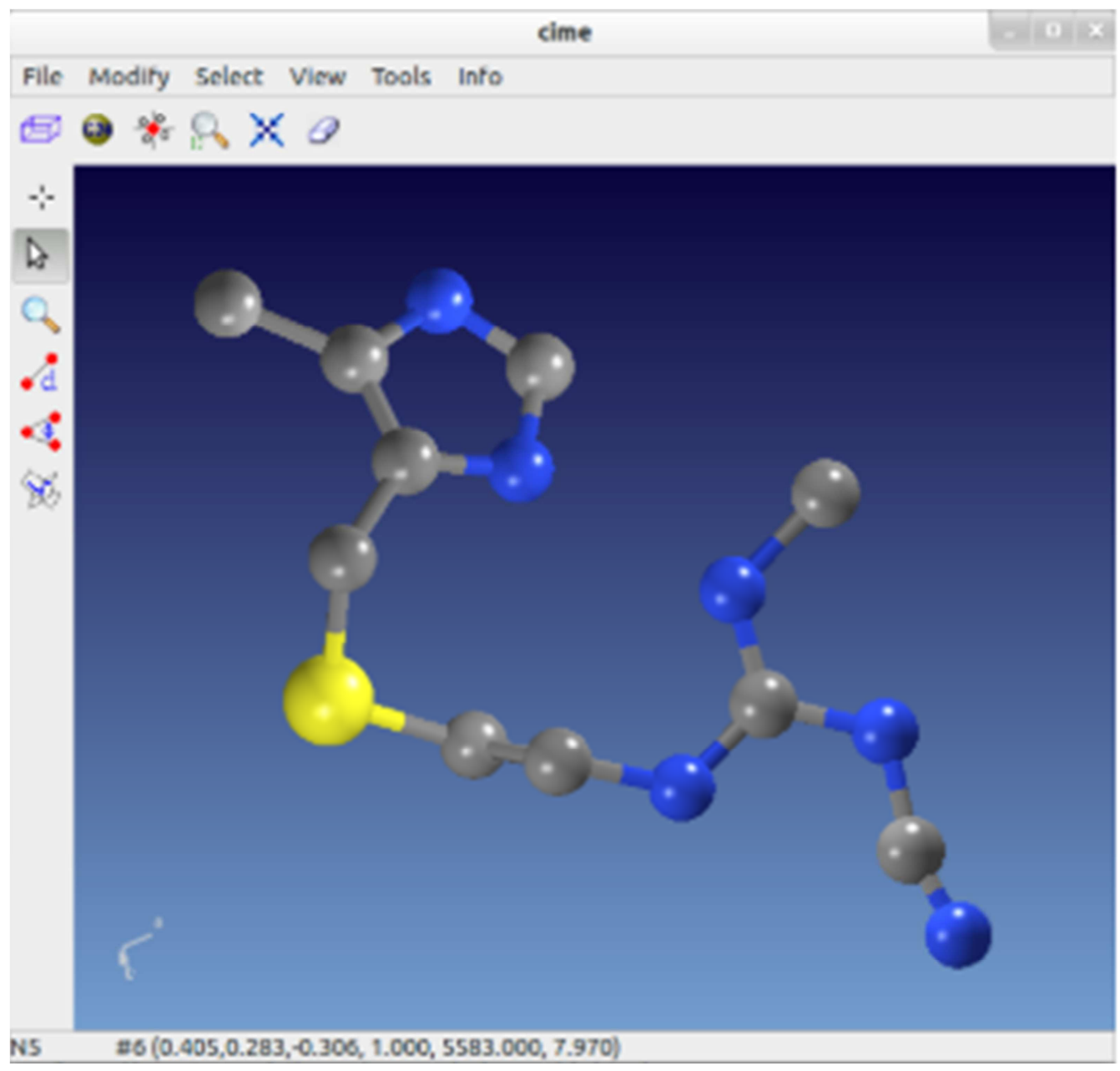

Figure 4. Crystal structure of example 2.

\section{Conclusion}

Reciprocal space methods for structure solution, of organic and inorganic compounds, is a straightforward tool for $a b$ initio phasing from powder diffraction data. The general limitation of RS methods is their linking on the quality of diffraction pattern, particularly, peak overlapping and experimental resolution. RS methods have the principal advantage of starting: diffraction data, the unit cell parameters, the chemical formula and the space group. The solving crystal structure is complex for polycrystalline powder. The big progress, which is noticed to be reached, particularly, in terms of software packages with computing performance and sophisticated graphical, is available to automatically solution process, from the determination of the unit cell parameters to structure validation (EXPO is an example). 


\section{References}

[1] J. W. Visser, J. Appl. Crystallogr., 1969, 2, 89.

[2] P. -E. Werner, L. Eriksson and M. Westdahl, J. Appl. Crystallogr., 1985, 18, 367.

[3] A. Boultif and D. Loue"r, J. Appl. Crystallogr., 1991, 24, 987.

[4] Altomare, A., Cuocci, C., Giacovazzo, C., Moliterni, A., Rizzi, R. (2009). Acta Cryst. A65, 183189.

[5] Rietveld, H. M. (1969). J. Appl. Cryst. 2, 65-71.

[6] A. Le Bail, H. Duroy and J. L. Fourquet, Mater. Res. Bull., 1988, 23, 447.

[7] G. S. Pawley, J. Appl. Crystallogr., 1981, 14, 357.

[8] Giacovazzo, C. (1998). IUCr /Oxford University Press, Oxford.

[9] Patterson, A. L. (1934). Phys. Rev. 46, 372-376.

[10] Bricogne, G. (1991). ActaCryst. A47, 803-829.

[11] Altomare A. Cuocci C. Moliterni A. and Rizzi R. (2019) International Tables for Crystallography Vol. H, Chapter 4. 2, pp. 395-413.

[12] Wilson A. J. C. (1942). London, Nature, 150, 151-152.

[13] Giacovazzo, C. (1977). ActaCryst. A33, 933-944.

[14] Giacovazzo, C. (1980). ActaCryst. A36, 362-372.

[15] Giacovazzo, C. (2001). International Tables for Crystallography, Volume B, pp. 210-234. Dordrecht: IUCr/Kluwer Academic Publishers.

[16] Giacovazzo, C. (2013). Phasing in Crystallography: A Modern Perspective. Oxford: IUCr/ Oxford University Press.

[17] CochranW. Acta Cryst 1955; 8: 473-8.

[18] Cascarano, G., Giacovazzo, C., Camalli, M., Spagna, R., Burla, M. C., Nunzi, A. \&Polidori, G. (1984). ActaCryst. A40, 278-283

[19] Karle, J., Hauptman, H. A. (1956). Acta Cryst. 9, 635-651.
[20] Altomare, A., Cuocci, C., Giacovazzo, C., Moliterni, A. \&Rizzi, R. (2011a). J. Appl. Cryst. 44, 448-453.

[21] Altomare, A., Caliandro, R., Giacovazzo, C., Moliterni, A. G. G., Rizzi, R. (2003). J. Appl. Cryst. 36, 230-238.

[22] Altomare, A., Caliandro, R., Cuocci, C., daSilva, I., Giacovazzo, C., Moliterni, A. G. G. \&Rizzi, R. (2004). J. Appl. Cryst. 37, 204-209.

[23] Harker, D. (1936). J. Chem. Phys. 4, 381-390.

[24] Estermann, M. A. \& David, W. I. F. (2002). Structure Determination from Powder Diffraction Data, pp. 202-218. Oxford University Press.

[25] Burla, M. C., Caliandro, R., Carrozzini, B., Cascarano, G. L., DeCaro, L., Giacovazzo, C., Polidori, G. \&Siliqi, D. (2007). J. Appl. Cryst. 40, 834-840.

[26] David, W. I. F. (1987). J. Appl. Cryst. 20, 316-319.

[27] Estermann, M. A., McCusker, L. B. \&Baerlocher, C. (1992). J. Appl. Cryst. 25, 539-543.

[28] Gilmore, C. J. (1996). ActaCryst. A52, 561-589.

[29] Gilmore, C. J., Henderson, K. \&Bricogne, G. (1991). ActaCryst. A47, 830-841.

[30] Magdysyuk, O. V. van Smaalen S. and Dinnebier R. E. (2019). International Tables for Crystallography Vol. H, Chapter 4. 8, pp. 473-488.

[31] AIT Mouha M. et al 2020 IOP Conf. Ser.: Mater. Sci. Eng. 783012005

[32] Altomare, A., Cuocci, C., Giacovazzo, C., Moliterni, A., Rizzi, R., Corriero, N., Falcicchio, A. (2013). J. Appl. Cryst., 46, 1231-1235.

[33] Altomare, A., Camalli, M., Cuocci, C., Giacovazzo, C., Moliterni, A. \&Rizzi, R. (2009). J. Appl. Cryst. 42, 1197-1202.

[34] Florence AJ, Shankl and N, Shankl and K et al. J. Appl. Cryst. 2005; 38: 249-59.

[35] Caliandro, R., Carrozzini, B., Cascarano, G. L., DeCaro, L., Giacovazzo, C. \&Siliqi, D. 2005a. ActaCryst. D61, 1080-87.

[36] Cernik, R. J., Cheetham, A. K., Prout, C. K., Watkin, D. J., Wilkinson, A. P., Willis, B. T. M. (1991). J. Appl. Cryst. 24, 222-226. 